\title{
Growth and development responses of some legume species inoculated with a mycorrhiza-based biofertilizer
}

\author{
N. Djébali ${ }^{1 *}$, S. Turki ${ }^{2}$, M. Zid ${ }^{2}$ and M. R. Hajlaoui ${ }^{2}$ \\ ${ }^{1}$ Laboratory of Legumes, Centre of Biotechnology of Borj Cedria, B.P. 901, 2050 Hammam- \\ Lif, Tunisia; \\ ${ }^{2}$ Laboratory of Plant Protection, the National Institute for Agricultural Research, INRA \\ Tunisia, Rue Hedi Karray, 2049 Ariana, Tunisia. \\ * Corresponding Author E-mail: dnaceur@yahoo.fr
}

\begin{abstract}
The application of beneficial microorganisms can enhance the growth of crops and increase their yields. In this context, we analysed the growth and development responses of six legume species inoculated by the mycorrhiza-based bio-fertilizer "Stanes Symbion ${ }^{\circledR}$ VAM Plus" in greenhouse conditions. The effect of inoculation by Symbion VAM on legumes growth and development depends on the species and the cultivar used. A significant increase of shoot fresh weight, number of nodules and root dry weight was observed in common bean, pea and alfalfa, respectively. The most positive effect of inoculation was observed with the A17 Medicago truncatula line, which showed an increase in shoot and root dry weights, in nodule and pod numbers, and an early blooming. However, chickpea and lentil inoculated plants didn't show any increase for all measured growth and development traits. This positive effect of inoculation on A17 line by the Symbion VAM biofertilizer was associated with important mycorrhiza root colonization (21 to $40 \%$ ) in comparison to the other legume cultivars at 50 days post inoculation. This study suggests that the success of inoculation with mycorrhiza-based inoculants depends on several factors including the density of inoculants in infective propagules and the length of legume life cycle.
\end{abstract}

Keywords: Fabaceae, Inoculation, Gigaspora, Glomus, Root colonization.

\section{INTRODUCTION}

Crop production of quality implies that the finished products are of high range and the use of technologies that better protect the environment. Therefore, any approach to reduce the utilisation of pesticides and chemical fertilizers and that protect crops and soil quality should be used. The symbiotic association between soil fungi and roots (mycorrhiza) of more than $90 \%$ of terrestrial plants constitutes the key for building durable agro-ecosystems. Of the seven types of mycorrhizae described in current scientific literature (arbuscular, ecto, ectendo, arbutoid, monotropoid, ericoid and orchidaceous mycorrhizae), the arbuscular and ectomycorrhizae are the most abundant and widespread (Siddiqui and Pichtel, 2008). The Vesicular Arbuscular Mycorrhiza (VAM) fungi, grouped in the phylum Glomeromycota, are the commonest mycorrhizal type involved in agricultural systems (Bethlenfalvay, 1992). To the well-known positive impacts of VAM on plant yields should be added several other benefits such as a better survival rate of colonized plants, the maintenance of plant biodiversity, the improvement of soil microflora (Boer et al., 2005), the resistance to biotic (Bødker et al., 2002; Dalpé, 2005) and abiotic environmental stresses (Evelin et al., 2009; Neumann and George, 2009), the improvement of soil structure and the reduction of pesticide use (Strack et al., 2003). Since VAM symbiosis can benefit plant growth and health, there is an increasing interest in their application and management, particularly within the context of sustainable agriculture. Therefore, the objective of this work was to analyze the effect of mycorrhiza-based biofertilizer on the growth and development of some large-grain and forage legumes grown in Tunisia.

\section{MATERIALS AND METHODS}

Plant Material: Six legumes species were used in this work: two cultivars (cv.) of pea (Pisum sativum) Rahma and Lincoln, the Chetoui cv. of chickpea (Cicer arietinum), a local lentil (Lens culinaris) cultivar, the Royal Nell cv. of common bean (Phaseolus vulgaris), the Gabès cv. of alfalfa (Medicago sativa) and the two lines of Medicago truncatula F83005.5 and A17. Disinfection of large- 
grain legume seeds (pea, chickpea, lentil and common bean) was done by incubation in $95^{\circ}$ alcohol for $30 \mathrm{~s}$, then in $0.2 \% \mathrm{HgCl}_{2}$ for 2 min. Seeds of the forage legumes ( $M$. sativa and $M$. truncatula) were disinfected and scarified in sulphuric acid $37 \%$ for 3 min for $M$. sativa and 6 min for M. truncatula. After disinfection, the legume seeds were washed 5 times with sterile distilled water and then kept in water until imbibition. The imbibed seeds were placed in sterile Petri dishes on moist filter paper. For large-grain legumes, the plates were incubated for $48 \mathrm{~h}$ at $25^{\circ} \mathrm{C}$ in the dark until seed germination. The seeds of forage legumes were putted for $48 \mathrm{~h}$ at $4^{\circ} \mathrm{C}$ and then transferred overnight at $25^{\circ} \mathrm{C}$ for germination.

Fungal material : Stanes Symbion ${ }^{\circledR}$ VAM Plus is a formulated product containing biologically active bioinoculums in an inert carrier material. Symbion VAM contains a combination of different species of mycorrhizae fungi (Glomus fasciculatum and
Gigaspora sp.) and the beneficial soil bacteria (solubilising phosphorus) belonging to the species Bacillus megaterium. Symbion VAM product contains $2.10^{2}$ infective propagules per $1 \mathrm{~kg}$.

Planting, inoculation and plant growth monitoring: Germinated seeds were transplanted into $500 \mathrm{ml}$ pots on a non sterilized clay garden soil (3.2\% organic mater, 2.48 ppm $\mathrm{P}_{2} \mathrm{O}_{5}, 2360.8 \mathrm{ppm}$ $\mathrm{K}_{2} \mathrm{O}$ ). The plants were grown under a plastic tunnel greenhouse. The inoculation of plants with Symbion VAM was done by placing $25 \mathrm{~g}$ of the product in contact of the roots of 15 days old seedlings. The control seedling plants did not receive inoculums. Irrigation of plants was made twice a week by putting the same amount of water for control and inoculated plants. Plant growth and development measurements were done every 15 days on inoculated and control plants until 50 days post inoculation. The measured traits are indicated in the Table 1.

Table 1. Measured growth and development traits and their abbreviations.

\begin{tabular}{|l|l|c|}
\hline \multirow{5}{*}{ Growth } & Trait & Abbreviation \\
\hline \multirow{5}{*}{} & Number of stems & NS \\
\cline { 2 - 3 } & Length of stems & NIN \\
\cline { 2 - 3 } & Number of internodes & NL \\
\cline { 2 - 3 } & Number of leaves & NF \\
\cline { 2 - 3 } & Number of flowers & NP \\
\cline { 2 - 3 } & Number of pods & WP \\
\cline { 2 - 3 } & Weight of pods & LR \\
\cline { 2 - 3 } & Length of roots & NN \\
\cline { 2 - 3 } & Number of nodules & SFW \\
\cline { 2 - 3 } & Shoot fresh weight & RFW \\
\cline { 2 - 3 } & Root fresh weight & SDW \\
\cline { 2 - 3 } & Shoot dry weight & RDW \\
\cline { 2 - 3 } & Root dry weight & D1F \\
\hline Development & Date of appearance of the first flower & D1P \\
\cline { 2 - 3 } & Date of appearance of the first pod & \\
\hline
\end{tabular}

Mycorrhiza structures staining and evaluation method of root colonization: The coloration of mycorrhiza structures in legume roots was made as described by Brundrett et al. (1984). The harvested roots were washed under tap water and conserved in ethanol $70 \%$ until use. The conserved roots were washed with sterile distilled water 5 times to remove ethanol and then cut into 1 to $2 \mathrm{~cm}$ pieces. The roots were blanched in $\mathrm{KOH}(10 \%)$ at $95^{\circ} \mathrm{C}$ for 15 minutes, and then rinsed 5 times with distilled water for 5 min. The coloration of roots was done with black ink
(Waterman) at $95^{\circ} \mathrm{C}$ for $10 \mathrm{~min}$. Excess of ink was removed by rinsing root pieces 5 times with distilled water. Finally the roots pieces were placed in a glycerol (50\%) and lactic acid (1\%) solution. Using a variation of the gridline intersection method as described in Giovannetti and Mosse (1980), the total root length colonization with intraradical structures was calculated from their presence in longitudinal microscopic intersections using a $20 \times$ magnification in a stereo binocular microscope. For each biological 
replicate, a minimum of 100 root segments was analyzed.

Statistical analyses: Data were subjected to an analysis of variance (ANOVA) using the Statistica software version 5.1 (www.statsoft.com) and the means of measured traits were compared using the LSD test ( $p \leq 0.05)$. Correlations between measured traits were estimated by computing the Pearson correlation coefficient $(r)$. Ten replicates were considered for each legume species.

\section{RESULTS AND DISCUSSION}

\section{Correlations analysis}

Among the 196 possible correlations, there are 38 significant and 36 are positive (Table 2). The total number of stems per plant (NS) is positively correlated with the number of leaves (NL), the total number of internodes (NIN), the shoot dry weight (SDW) and the root dry weight (RDW) (Table 2). This finding suggests that the plants with large stem number exhibit more leaves and internodes and they have a well developed shoot and root systems. However, NS was negatively correlated with the flowering time (D1F) and the number of flowers per plant (NF). This result indicates that the plants showing high number of ramifications are those that bloom early. Accordingly, similar results were reported by Badri et al. (2007; 2008a; 2008b) and Arraouadi et al. (2009) studying the growth and development of several $M$. truncatula, $M$. laciniata and $M$. ciliaris populations collected from different Tunisian regions.

Table 2. Correlations between different growth and development traits measured on six legumes inoculated or not by the biofertilizer "Stanes Symbion ${ }^{\circledR}$ VAM Plus".

\begin{tabular}{|c|c|c|c|c|c|c|c|c|c|c|c|c|c|}
\hline & NS & LS & $\mathrm{NL}$ & NIN & D1F & NF & D1P & NP & SFW & SDW & RFW & RDW & NN \\
\hline NS & 1 & & & & & & & & & & & & \\
\hline LS & $0.12 \mathrm{~ns}$ & 1 & & & & & & & & & & & \\
\hline $\mathrm{NL}$ & $0.41^{* * *}$ & $0.62^{* * *}$ & 1 & & & & & & & & & & \\
\hline NIN & $0.31^{* *}$ & $0.64^{* * *}$ & $0.95^{* * *}$ & 1 & & & & & & & & & \\
\hline $\mathrm{D} 1 \mathrm{~F}$ & -0.23 * & 0.33 ** & $-0.07 \mathrm{~ns}$ & $-0.01 \mathrm{~ns}$ & 1 & & & & & & & & \\
\hline NF & -0.25 * & $0.39^{* * *}$ & $0.02 \mathrm{~ns}$ & $0.07 \mathrm{~ns}$ & $0.82^{* * *}$ & 1 & & & & & & & \\
\hline D1P & $-0.17 \mathrm{~ns}$ & $0.09 \mathrm{~ns}$ & $-0.17 \mathrm{~ns}$ & $-0.11 \mathrm{~ns}$ & $0.72^{* * *}$ & $0.55^{* * *}$ & 1 & & & & & & \\
\hline $\mathrm{NP}$ & $0.06 \mathrm{~ns}$ & $0.001 \mathrm{~ns}$ & $-0.15 n s$ & $-0.11 \mathrm{~ns}$ & $0.56^{* * *}$ & $0.36^{* *}$ & $0.78^{* * *}$ & 1 & & & & & \\
\hline SFW & $0.11 \mathrm{~ns}$ & $0.19 \mathrm{~ns}$ & $0.01 \mathrm{~ns}$ & $0.02 \mathrm{~ns}$ & $0.61^{* * *}$ & $0.46^{* * *}$ & $0.65^{* * *}$ & $0.66^{* * *}$ & 1 & & & & \\
\hline SDW & 0.25 * & $0.5^{* * *}$ & $0.21 \mathrm{~ns}$ & $0.20 \mathrm{~ns}$ & $0.52^{* * *}$ & $0.41^{* * *}$ & $0.40^{* * *}$ & $0.42^{* * *}$ & $0.64^{* * *}$ & 1 & & & \\
\hline RFW & $0.51^{* * *}$ & $0.02 \mathrm{~ns}$ & $0.09 \mathrm{~ns}$ & $0.012 \mathrm{~ns}$ & $0.12 \mathrm{~ns}$ & $0.04 \mathrm{~ns}$ & $0.13 \mathrm{~ns}$ & $0.37^{* *}$ & $0.44^{* * *}$ & $0.57^{* * *}$ & 1 & & \\
\hline RDW & 0.32 ** & $0.03 \mathrm{~ns}$ & $0.1 \mathrm{~ns}$ & $0.002 \mathrm{~ns}$ & $0.02 \mathrm{~ns}$ & $-0.06 \mathrm{~ns}$ & $-0.04 n s$ & $0.13 \mathrm{~ns}$ & 0.31 ** & $0.50^{* * *}$ & $0.87^{* * *}$ & 1 & \\
\hline NN & $0.09 \mathrm{~ns}$ & $0.18 \mathrm{~ns}$ & $0.02 \mathrm{~ns}$ & $0.01 \mathrm{~ns}$ & $0.20 \mathrm{~ns}$ & $0.19 \mathrm{~ns}$ & $0.11 \mathrm{~ns}$ & 0.26 * & $0.31^{* *}$ & $0.37^{\text {** }}$ & 0.27 * & 0.24 * & 1 \\
\hline
\end{tabular}

ns: not significant $(p>0.05),{ }^{*}: p \leq 0.01,{ }^{* *}: p \leq 0.001,{ }^{* * *}: p \leq 0.001$

The total length of stems (LS) was positively correlated with NF, NIN and SDW. By contrast to NS, the length of stems (LS) is positively correlated with flowering time (D1F) and the number of flowers (NF).Thus, it seems that plants with important growth are those that bloom late and give a large number of flowers. This is quite logical, because during the early stages of growth, when conditions are favourable, energy produced by plants is devoted to growth depends on the flowering time (Badri et al., 2007). This finding is reinforced by the fact that there is a positive correlation between the number of days that bring the plant to flower (D1F) and the SDW. D1F is also positively correlated with the number of flowers (NF), number of pods (NP) and the date of apparition of the first pod (D1P). Laouar and Abdelguerfi (1999) reported a positive correlation between flowering time 
and pods yield. Indeed, as shown above, a welldeveloped plant produces more flowers and pods, since the number of flowers (NF) is proportional to plant biomass. This is confirmed by the positive correlation between NF and SDW.

The number of nodules (NN) is positively correlated with the RFW and RDW. This finding indicates that more the legume root system is developed, more it contains nodules. A positive correlation was also observed between NN and SFW, SDW and NP, indicating that nodulation positively influence plant biomass and pods production. This shows the positive effect of nodulation on growth and development of plants through the nitrogen biological fixation (Aouani et al., 2001; Santalla et al., 2001; Badri et al., 2010).

Effect of mycorrhiza inoculation on legume growth and development: The effect of inoculation by Symbion VAM on legumes growth and development depends on the species and cultivar factors. In fact, at the species level we observed a positive effect of mycorrhiza inoculation in pea, common bean, alfalfa and $M$. truncatula for at least one trait (Table 3). However, no significant positive effect was observed for all measured parameters for chickpea and lentil (Table 3). At the cultivar level, a significant increase in nodule number (NN) was observed for the cv. Lincoln, but no such increase was observed in the cv. Rahma. Moreover, a difference between the two $M$. truncatula lines was observed in terms of their reaction to the inoculation by Symbion VAM. Indeed, a significant increase was noted in the A17 inoculated plants for several traits corresponding to NS, LS, D1F, D1P, NF, NP, SDW, RFW, RDW and NN. However, no significant increase was observed for all measured traits in F83005.5 line.

Table 3. Comparison of means of different growth and development traits measured on six legume species inoculated or not by the biofertilizer "Stanes Symbion ${ }^{\circledR}$ VAM Plus".

\begin{tabular}{|c|c|c|c|c|c|c|c|c|c|c|c|c|c|c|}
\hline $\begin{array}{l}\text { Legume } \\
\text { species } \\
\text { (common } \\
\text { name) }\end{array}$ & $\begin{array}{l}\text { Cultivar- } \\
\text { treatment }\end{array}$ & NS & LS & NL & NIN & D1F & NF & D1P & NP & SFW & $\begin{array}{l}\text { SD } \\
\text { W }\end{array}$ & RFW & $\begin{array}{l}\text { RD } \\
W\end{array}$ & NN \\
\hline \multirow{2}{*}{$\begin{array}{l}\text { Cicer } \\
\text { arietinum } \\
\text { (ckickpea) }\end{array}$} & $\begin{array}{l}\text { Chetoui } \\
\text { inoculated }\end{array}$ & $\begin{array}{l}2.75 \\
\text { bcde }\end{array}$ & $\begin{array}{l}34.1 \\
\text { bcd }\end{array}$ & $\begin{array}{c}24 \\
a\end{array}$ & $\begin{array}{c}25.5 \\
a\end{array}$ & nd & $\begin{array}{c}0 \\
\mathrm{~cd}\end{array}$ & nd & $\begin{array}{l}0 \\
d\end{array}$ & $\begin{array}{l}2.08 \\
\text { efg }\end{array}$ & $\begin{array}{l}0.75 \\
\text { de }\end{array}$ & $\begin{array}{c}3.62 \\
\mathrm{~cd}\end{array}$ & $\begin{array}{c}0.42 \\
\mathrm{c}\end{array}$ & $\begin{array}{l}0 \\
\mathrm{C}\end{array}$ \\
\hline & $\begin{array}{l}\text { Chetoui } \\
\text { control }\end{array}$ & $\begin{array}{l}2.5 \\
\text { cde }\end{array}$ & $\begin{array}{c}25.70 \\
\text { cdef }\end{array}$ & $\begin{array}{c}22.75 \\
a b\end{array}$ & $\begin{array}{c}20.25 \\
a b\end{array}$ & nd & $\begin{array}{c}0 \\
\mathrm{~cd}\end{array}$ & nd & $\begin{array}{l}0 \\
d\end{array}$ & $\begin{array}{c}1.39 \\
\mathrm{~g}\end{array}$ & $\begin{array}{c}0.41 \\
\mathrm{e}\end{array}$ & $\begin{array}{l}2.69 \\
\text { cde }\end{array}$ & $\begin{array}{l}0.3 \\
\mathrm{~cd}\end{array}$ & $\begin{array}{l}0 \\
\mathrm{C}\end{array}$ \\
\hline \multirow{4}{*}{$\begin{array}{l}\text { Pisum } \\
\text { sativum } \\
\text { (pea) }\end{array}$} & $\begin{array}{l}\text { Rahma } \\
\text { inoculated }\end{array}$ & $\begin{array}{c}1.83 \\
\text { ef }\end{array}$ & $\begin{array}{c}69.33 \\
a\end{array}$ & $\begin{array}{l}21 \\
a b\end{array}$ & $\begin{array}{c}19.75 \\
b\end{array}$ & $\begin{array}{c}36.25 \\
a b\end{array}$ & $\begin{array}{c}1.33 \\
a b\end{array}$ & $\begin{array}{c}14.5 \\
\mathrm{bc}\end{array}$ & $\begin{array}{c}0.92 \\
\mathrm{~cd}\end{array}$ & $\begin{array}{c}4.24 \\
\mathrm{~cd}\end{array}$ & $\begin{array}{c}1.04 \\
\mathrm{~cd}\end{array}$ & $\begin{array}{l}2.68 \\
\text { cde }\end{array}$ & $\begin{array}{c}0.29 \\
\mathrm{~cd}\end{array}$ & $\begin{array}{c}20.33 \\
a b\end{array}$ \\
\hline & $\begin{array}{l}\text { Rahma } \\
\text { control }\end{array}$ & $\begin{array}{c}1.83 \\
\text { ef }\end{array}$ & $\begin{array}{c}66.62 \\
a\end{array}$ & $\begin{array}{c}19 \\
a b c\end{array}$ & $\begin{array}{c}17.92 \\
b\end{array}$ & $\begin{array}{c}36.5 \\
a b\end{array}$ & $\begin{array}{l}1.5 \\
a b\end{array}$ & $\begin{array}{c}24.67 \\
\text { C }\end{array}$ & $\begin{array}{c}1.17 \\
\mathrm{~cd}\end{array}$ & $\begin{array}{c}4.32 \\
\mathrm{~cd}\end{array}$ & $\begin{array}{l}1.3 \\
b c\end{array}$ & $\begin{array}{c}2.14 \\
\text { def }\end{array}$ & $\begin{array}{l}0.3 \\
\mathrm{~cd}\end{array}$ & $\begin{array}{c}12.33 \\
a b c\end{array}$ \\
\hline & $\begin{array}{l}\text { Lincoln } \\
\text { inoculated }\end{array}$ & $\begin{array}{c}1.25 \\
f\end{array}$ & $\begin{array}{c}23.23 \\
\text { def }\end{array}$ & $\begin{array}{c}12.75 \\
\mathrm{~cd}\end{array}$ & $\begin{array}{c}11.38 \\
\mathrm{c}\end{array}$ & $\begin{array}{c}50.5 \\
a\end{array}$ & $\begin{array}{c}1.88 \\
a\end{array}$ & $\begin{array}{c}47.75 \\
a\end{array}$ & $\begin{array}{c}4.5 \\
b\end{array}$ & $\begin{array}{l}6.5 \\
a b\end{array}$ & $\begin{array}{c}0.92 \\
\mathrm{~cd}\end{array}$ & $\begin{array}{c}3.63 \\
\mathrm{C}\end{array}$ & $\begin{array}{c}0.44 \\
\mathrm{C}\end{array}$ & $\begin{array}{c}27.13 \\
a\end{array}$ \\
\hline & $\begin{array}{l}\text { Lincoln } \\
\text { control }\end{array}$ & $\begin{array}{c}1.00 \\
f\end{array}$ & $\begin{array}{c}21.52 \\
\text { def }\end{array}$ & $\begin{array}{l}10 \\
\text { de }\end{array}$ & $\begin{array}{c}9 \\
\mathrm{~cd}\end{array}$ & $\begin{array}{c}50.63 \\
a\end{array}$ & $\begin{array}{l}1.25 \\
a b c\end{array}$ & $\begin{array}{c}55.25 \\
a\end{array}$ & $\begin{array}{c}2.88 \\
\text { bc }\end{array}$ & $\begin{array}{l}5.27 \\
\text { bcde }\end{array}$ & $\begin{array}{c}0.91 \\
\text { cd }\end{array}$ & $\begin{array}{l}2.26 \\
\text { cdef }\end{array}$ & $\begin{array}{l}0.2 \\
\mathrm{~cd}\end{array}$ & $\begin{array}{c}6.13 \\
\text { bc }\end{array}$ \\
\hline \multirow{2}{*}{$\begin{array}{l}\text { Lens } \\
\text { culinaris } \\
\text { (lentil) }\end{array}$} & $\begin{array}{l}\text { Local cV. } \\
\text { inoculated }\end{array}$ & $\begin{array}{c}3.66 \\
b\end{array}$ & $\begin{array}{c}48.15 \\
b\end{array}$ & $\begin{array}{c}23.5 \\
a\end{array}$ & $\begin{array}{c}21.17 \\
a b\end{array}$ & nd & $\begin{array}{c}0 \\
\mathrm{~cd}\end{array}$ & nd & $\begin{array}{c}0 \\
\mathrm{~cd}\end{array}$ & $\begin{array}{c}1.88 \\
\mathrm{~g}\end{array}$ & $\begin{array}{c}0.47 \\
\mathrm{e}\end{array}$ & $\begin{array}{c}1.62 \\
\text { ef }\end{array}$ & $\begin{array}{c}0.19 \\
\mathrm{~cd}\end{array}$ & $\begin{array}{l}5.5 \\
b c\end{array}$ \\
\hline & $\begin{array}{l}\text { Local } \quad \mathrm{cV} \text {. } \\
\text { control }\end{array}$ & $\begin{array}{l}3.5 \\
\mathrm{bc}\end{array}$ & $\begin{array}{l}37.1 \\
\text { bcd }\end{array}$ & $\begin{array}{c}24.33 \\
\mathrm{a}\end{array}$ & $\begin{array}{c}22.83 \\
a b\end{array}$ & nd & $\begin{array}{c}0 \\
\mathrm{~cd}\end{array}$ & nd & $\begin{array}{c}0 \\
\mathrm{~cd}\end{array}$ & $\begin{array}{l}3.26 \\
\text { defg }\end{array}$ & $\begin{array}{c}0.50 \\
\mathrm{e}\end{array}$ & $\begin{array}{c}1.39 \\
\text { ef }\end{array}$ & $\begin{array}{c}0.22 \\
\mathrm{~cd}\end{array}$ & $\begin{array}{c}3.17 \\
\mathrm{bc}\end{array}$ \\
\hline \multirow{2}{*}{$\begin{array}{l}\text { Phaseolus } \\
\text { vulgaris } \\
\text { (common } \\
\text { bean) }\end{array}$} & $\begin{array}{l}\text { Royal Nell } \\
\text { inoculated }\end{array}$ & $\begin{array}{l}1.5 \\
\text { ef }\end{array}$ & $\begin{array}{c}8.25 \\
\text { ef }\end{array}$ & $\begin{array}{c}4.5 \\
\text { ef }\end{array}$ & $\begin{array}{c}1.5 \\
\mathrm{e}\end{array}$ & nd & $\begin{array}{c}0 \\
\mathrm{~cd}\end{array}$ & nd & $\begin{array}{c}0 \\
\mathrm{~cd}\end{array}$ & $\begin{array}{l}5.11 \\
\text { bcde }\end{array}$ & $\begin{array}{c}0.62 \\
\text { de }\end{array}$ & $\begin{array}{c}1.11 \\
\text { ef }\end{array}$ & $\begin{array}{c}0.17 \\
\mathrm{~cd}\end{array}$ & $\begin{array}{c}0 \\
b c\end{array}$ \\
\hline & $\begin{array}{ll}\text { Royal } & \text { Nell } \\
\text { control } & \\
\end{array}$ & $\begin{array}{l}1 \\
\mathrm{f} \\
\end{array}$ & $\begin{array}{c}3.25 \\
f\end{array}$ & $\begin{array}{l}2 \\
f\end{array}$ & $\begin{array}{l}0 \\
\mathrm{e}\end{array}$ & nd & $\begin{array}{c}0 \\
\mathrm{~cd}\end{array}$ & nd & $\begin{array}{c}0 \\
\mathrm{~cd}\end{array}$ & $\begin{array}{c}0.65 \\
\mathrm{~g}\end{array}$ & $\begin{array}{c}0.32 \\
\mathrm{e}\end{array}$ & $\begin{array}{c}0.28 \\
f\end{array}$ & $\begin{array}{c}0.02 \\
\mathrm{~d}\end{array}$ & $\begin{array}{c}0 \\
b c\end{array}$ \\
\hline \multirow{2}{*}{$\begin{array}{l}\text { Medicago } \\
\text { sativa } \\
\text { (alfalfa) }\end{array}$} & $\begin{array}{l}\text { Gabès } \\
\text { inoculated }\end{array}$ & $\begin{array}{c}2.20 \\
\text { def }\end{array}$ & $\begin{array}{c}27.83 \\
\text { cde }\end{array}$ & $\begin{array}{l}15 \\
\mathrm{~cd}\end{array}$ & $\begin{array}{c}10.8 \\
\mathrm{~cd}\end{array}$ & 13.8 & $\begin{array}{l}0.2 \\
\text { bcd } \\
\end{array}$ & nd & $\begin{array}{c}0 \\
\mathrm{~cd}\end{array}$ & $\begin{array}{l}4.33 \\
\text { cdef }\end{array}$ & $\begin{array}{c}1.21 \\
\mathrm{bc}\end{array}$ & $\begin{array}{c}6.33 \\
b\end{array}$ & $\begin{array}{c}1.19 \\
b\end{array}$ & $\begin{array}{l}17.6 \\
\mathrm{abc}\end{array}$ \\
\hline & Gabès control & $\begin{array}{l}3.00 \\
\text { bcd }\end{array}$ & $\begin{array}{c}37.33 \\
\text { bcd }\end{array}$ & $\begin{array}{c}16 \\
\text { bcd }\end{array}$ & $\begin{array}{c}12.33 \\
\mathrm{c}\end{array}$ & nd & $\begin{array}{c}0 \\
\mathrm{~cd}\end{array}$ & nd & $\begin{array}{c}0 \\
\mathrm{~cd}\end{array}$ & $\begin{array}{c}3.52 \\
\text { cdefg }\end{array}$ & $\begin{array}{l}1.05 \\
\text { bcd }\end{array}$ & $\begin{array}{c}8.33 \\
b\end{array}$ & $\begin{array}{c}1.5 \\
\mathrm{a} \\
\end{array}$ & $\begin{array}{c}6 \\
b c\end{array}$ \\
\hline \multirow{4}{*}{$\begin{array}{l}\text { Medicago } \\
\text { truncatula } \\
\text { (annual } \\
\text { medic) }\end{array}$} & $\begin{array}{l}\text { A17 } \\
\text { inoculated }\end{array}$ & $\begin{array}{c}13.00 \\
a\end{array}$ & $\begin{array}{l}56.5 \\
a b c\end{array}$ & $\begin{array}{c}17 \\
\text { abcd }\end{array}$ & $14 \mathrm{bcd}$ & 58 & $\begin{array}{c}11.0 \\
a\end{array}$ & 62 & $\begin{array}{c}10.0 \\
a\end{array}$ & $\begin{array}{c}10.39 \\
a\end{array}$ & $\begin{array}{c}2.57 \\
a\end{array}$ & $\begin{array}{c}15.44 \\
a\end{array}$ & $\begin{array}{c}1.33 \\
a b\end{array}$ & $\begin{array}{c}51 \\
a\end{array}$ \\
\hline & A17 control & $\begin{array}{c}4.00 \\
\text { bc }\end{array}$ & $\begin{array}{c}13 \\
\text { def }\end{array}$ & $\begin{array}{c}11 \\
\text { cdef }\end{array}$ & $\begin{array}{c}8 \\
\text { cde } \\
\end{array}$ & nd & $\begin{array}{c}0 \\
\mathrm{~cd}\end{array}$ & nd & $\begin{array}{c}0 \\
\mathrm{~cd}\end{array}$ & $\begin{array}{l}6.17 \\
\text { abcd }\end{array}$ & $\begin{array}{l}1.21 \\
\text { bcd }\end{array}$ & $\begin{array}{l}2.36 \\
\text { cdef }\end{array}$ & $\begin{array}{c}0.28 \\
\mathrm{~cd}\end{array}$ & $\begin{array}{l}10 \\
\mathrm{bc}\end{array}$ \\
\hline & $\begin{array}{l}\text { F83005.5 } \\
\text { inoculated }\end{array}$ & $\begin{array}{l}3.00 \\
\text { bcd }\end{array}$ & $\begin{array}{c}6.83 \\
f\end{array}$ & $\begin{array}{c}9.33 \\
\text { def }\end{array}$ & $\begin{array}{c}5 \\
\text { de }\end{array}$ & nd & $\begin{array}{c}0 \\
\mathrm{~cd}\end{array}$ & nd & $\begin{array}{c}0 \\
\mathrm{~cd}\end{array}$ & $\begin{array}{c}1.88 \\
\mathrm{fg}\end{array}$ & $\begin{array}{c}0.45 \\
\mathrm{e}\end{array}$ & $\begin{array}{l}3.33 \\
\text { cde }\end{array}$ & $\begin{array}{c}0.31 \\
\mathrm{~cd}\end{array}$ & $\begin{array}{c}3 \\
b c\end{array}$ \\
\hline & $\begin{array}{l}\text { F83005.5 } \\
\text { control }\end{array}$ & $\begin{array}{l}3.00 \\
\text { bcd }\end{array}$ & $\begin{array}{c}6.33 \\
f\end{array}$ & $\begin{array}{l}9.67 \\
\text { def }\end{array}$ & $\begin{array}{l}6.33 \\
\text { cde }\end{array}$ & nd & $\begin{array}{c}0 \\
\mathrm{~cd}\end{array}$ & nd & $\begin{array}{c}0 \\
\mathrm{~cd}\end{array}$ & $\begin{array}{l}2.23 \\
\text { defg }\end{array}$ & $\begin{array}{c}0.51 \\
\mathrm{e}\end{array}$ & $\begin{array}{l}3.38 \\
\text { cde }\end{array}$ & $\begin{array}{c}0.33 \\
\mathrm{~cd}\end{array}$ & $\begin{array}{c}2 \\
\mathrm{Bc}\end{array}$ \\
\hline
\end{tabular}

Mean values followed by the same letter are not significantly different at $p \leq 0.05$ (LSD test). nd: not determined. 
The shoot part of A17 inoculated plants was more developed than in control plants, which is an interesting criterion as $M$. truncatula is a forage legume. Feddermann et al. (2008) showed enhanced A17 plant biomass ten weeks after inoculation with two Glomus species. The observed increase in nodule number in legumes (A17 and Lincoln) after inoculation with mycorrhiza is in agreement with previous finding which indicates that the association of mycorrhiza with rhizobia in field inoculation can improves significantly the nodulation (Barea, 1980).

Assessment of mycorrhiza colonization of legume roots: The Brundrett et al. (1984) coloration protocol was very sensitive and simple for the detection of mycorrhiza structures in the roots of the studied legumes (Figure 1A). Three different mycorrhiza structures were observed in the legume roots, which correspond to the intercellular hyphae (Figure 1B) and vesicles (Figure 1C), and the intracellular arbuscules (Figure 1D). Observation of the control roots showed no mycorrhiza structures for the six tested legumes at $50 \mathrm{dpi}$ (Table 4). This result indicates that the used soil does not contain native mycorrhizae species. Moreover, this finding proves that observed mycorrhiza structures in the roots of inoculated plants are due to the Symbion VAM inoculants.

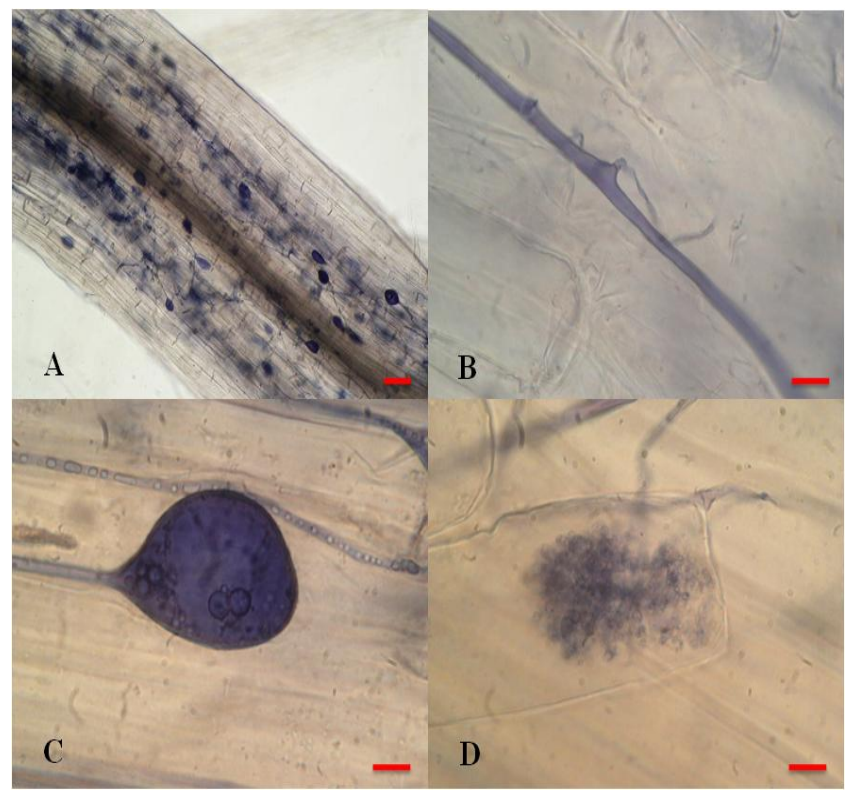

Fig 1. Mycorrhiza structures observed in legume roots at 50 days post inoculation with the biofertilizer "Stanes Symbion ${ }^{\circledR}$ VAM Plus". A: root with intraradical mycorrhiza structures $($ Bar $=\mathbf{3 0 0} \mu \mathrm{m}), \mathrm{B}$ : intercellular hypha, $C$ : intercellular vesicle, $D$ : intracellular arbuscule. B, C, D Bars $=50 \mu \mathrm{m}$.
Table 4. Percentage of mycorrhized legume plants at $\mathbf{5 0}$ days post inoculation with the biofertilizer "Stanes Symbion ${ }^{\circledR}$ VAM Plus".

\begin{tabular}{|c|c|c|}
\hline \multirow[t]{2}{*}{ Legume species (cultivar) } & \multicolumn{2}{|c|}{$\begin{array}{l}\text { Percentage of } \\
\text { mycorrhized } \\
\text { plants (\%) }\end{array}$} \\
\hline & Inoculated & Control \\
\hline Cicer arietinum (Chetoui) & 0 & 0 \\
\hline Pisum sativum (Rahma) & $60.6(+)$ & 0 \\
\hline Pisum sativum (Lincoln) & $\begin{array}{c}60.6(+) \\
33.4(++)\end{array}$ & 0 \\
\hline Lens culinaris (Local) & $100(+)$ & 0 \\
\hline Medicago sativa (Gabès) & $60.6(+)$ & 0 \\
\hline Medicago truncatula (A17) & $\begin{array}{c}60.6(++) \\
30.4(+)\end{array}$ & 0 \\
\hline Medicago truncatula (F83005.5) & $100(+)$ & 0 \\
\hline Phaseolus vulgaris (Royal Nell) & $100(+)$ & 0 \\
\hline
\end{tabular}

Percentage of the total root length colonized with

mycorrhizal structures: + (1 to $20 \%)$; ++ (21 to $40 \%)$.

The M. truncatula A17 line showed important mycorrhiza root colonization in comparison to the other legume species. This result is consistent with the significant increase in growth and development of the A17 inoculated plants in comparison to the control (Table 3). However, the line F83005.5 showed less root colonization by mycorrhiza structures in comparison to A17 line. Contrasted responses between these two $M$. truncatula lines were detected in interaction with several microorganisms including fungi (Ameline-Torregrosa et al., 2008), oomycete (Djébali et al., 2009) and bacteria (Vailleau et al., 2007). Therefore, by the use of $M$. truncatula genetic maps and recombinant inbred lines we can identify genetic components of traits determining mycorrhiza responsiveness. Moreover, the combination of genetic analyses with abundant genomic data (Young et al. 2005) allows identification of the molecular basis for mycorrhiza responsiveness in legumes.

The inoculated Chetoui plants (chickpea) did not show any mycorrhiza structures in their roots at 50 dpi. However, Al-Raddad (1991) showed that chickpea was mycorrhized by various Glomus species including $G$. fasciculatum. Pea, lentil, common bean and alfalfa showed low colonization of roots with mycorrhiza (Table 4), which can explain why we didn't find a positive effect of Symbion VAM inoculation for most measured growth and development traits on these legume species (Table 3). Two hypotheses can be made to explain the low colonization of roots observed in most legumes: (i) 
the low infective propagules concentration in the used inoculants, and (ii) the short life cycle of most large-grain legume species which showed growth slowdown beyond 30 dpi (data not shown); so they didn't profit well from association with mycorrhizae fungi. Some studies reported that the development of mycorrhizae fungi and their effects on plant growth are greater in soils with low nutrient content, in particular with low phosphorus (Baar, 2008). However, in our study despite the low phosphorus content in the used culture soil we have generally observed low root colonization in legume species.

\section{CONCLUSION}

This work shows that the effect of inoculation by Symbion VAM on legumes growth and development depends on the species and cultivar used. The success of inoculation with mycorrhiza-based biofertilizer on legumes depends on several factors including the density of inoculants in infective propagules and the length of legume life cycle.

\section{ACKNOWLEDGEMENTS}

We are grateful to Mr. Hedi Hamrouni (INRGREF, Tunisia) for soil analysis and Dr. Mounawer Badri (CBBC, Tunisia) for critical reading of the manuscript.

\section{REFERENCES}

Al-Raddad, A (1991). Response of bean, broadbean and chickpea plants to inoculation with Glomus species. Sci. Hort. 46:195-200.

Ameline-Torregrosa, C., M. Cazaux, D. Danesh, F. Chardon, S.B. Cannon, M.T. Esquerre-Tugaye, B. Dumas, N.D. Young, D.A. Samac, T. Huguet, and Jacquet C (2008). Genetic dissection of resistance to anthracnose and powdery mildew in Medicago truncatula. Mol. Plant-Microbe Interact. 21:61-69.

Aouani, M.E., R. Mhamdi, M. Jebara and Amarger N. (2001). Characterization of rhizobia nodulating chickpea in Tunisia. Agronomie 21:577-581.

Arraouadi, S., M. Badri, C. Abdul Jaleel, N. Djébali, H. Ilahi, T. Huguet and Aouani M.E (2009). Analysis of Genetic Variation in Natural Populations of Medicago truncatula of Southern Tunisian Ecological Areas. Using Morphological Traits and SSR Markers. Tropical. Plant Biol. 2:122-132.

Baar, J (2008). From production to application of arbuscular mycorrhizal Fungi in agricultural systems: requirements and needs, In: A. Varma (Ed). pp. 361373. Mycorrhiza. Springer.

Badri, M., A. Zitoun, H. Ilahi, T. Huguet, Aouani M.E (2008a). Morphological and microsatellite diversity associated with ecological factors in natural populations of Medicago laciniata Mill. (Fabaceae). J. Genet. 87:241-255.

Badri, M., A. Zitoun, S. Soula, H. Ilahi, T. Huguet and Aouani M.E (2008b). Low levels of quantitative and molecular genetics differentiation among natural populations of Medicago ciliaris Kroch. (Fabaceae) of different Tunisian eco-geographical origin. Conser. Genet. 9:1509-1520.

Badri, M., H. llahi, T. Huguet, and Aouani M.E (2007). Quantitative and molecular genetic variation in sympatric populations of Medicago laciniata and $M$. truncatula (Fabaceae): relationships with ecogeographical factors. Genet. Res. Camb. 89:107-122.

Badri, M., S. Soula, T. Huguet and Aouani M.E (2010). Symbiotic performance of Medicago ciliaris lines in association with different Sinorhizobium strains. J. Ecobiotech. 2:6-11.

Barea, J.M., J.L. Escudero, G. Azcon and de Aguilar C (1980). Effects of introduced and indigenous VA mycorrhizal fungi on nodulation, growth and nutrition of Medicago sativa in phosphate-fixing soils as affected by P fertilizers. Plant Soil 54:283-296.

Bethlenfalvay, G.J. 1992. Mycorrhizae in the agricultural plant-soil system. Symbiosis 14:413-425.

Bødker, L., R. Kjøller, K. Kristensen, and Rosendahl S (2002). Interactions between indigenous arbuscular mycorrhizal fungi and Aphanomyces euteiches in fieldgrown pea. Mycorrhiza 12:7-12.

Boer, W., L.B. Folman, R.C. Summerbell and Boddy L (2005). Living in a fungal world: Impact of fungi on soil bacterial niche development. FEMS Microbiol. Rev. 29:795-811.

Brundrett, M.C., Y. Piché, and Peterson R.L (1984). A new method for observing the morphology of vesiculararbuscular mycorrhizae. Can. J. Bot. 63:2128-2134.

Dalpé, Y (2005). Les mycorhizes: un outil de protection des plantes mais non une panacée. Phytoprotection 86:5359.

Djébali, N., A. Jauneau, C. Ameline-Torregrosa, F. Chardon, V. Jaulneau, C. Mathé, A. Bottin, M. Cazaux, M. L. Pilet-Nayel, A. Baranger, M. E. Aouani, M. T. Esquerré-Tugayé, B. Dumas, T. Huguet, and Jacquet C (2009). Partial resistance of Medicago truncatula to Aphanomyces euteiches is associated with protection of the root stele and is controlled by a major QTL rich in proteasome-related genes. Mol. Plant Microbe Interact. 22:1043-1055.

Evelin, H., R. Kapoor and Giri B (2009). Arbuscular mycorrhizal fungi in alleviation of salt stress. Ann. Bot. 104: $1263-1280$.

Feddermann, N., T. Boller, P. Salzer, S. Elfstrand, A. Wiemken, and Elfstrand M (2008). Medicago 
truncatula shows distinct patterns of mycorrhizarelated gene expression after inoculation with three different arbuscular mycorrhizal fungi. Planta 227:671680.

Giovannetti M, and Mosse B. (1980). An evaluation of techniques for measuring vesicular arbuscular mycorrhizal infection in roots. New Phytol. 84:489-500.

Laouar, M. and Abdelguerfi A (1999). Variability in pod and seed production in some spontaneous Medicago intertexta populations. Cah. Opt. Méditerr.39:111-117.

Neumann, E., and George E (2009). The effect of arbuscular mycorrhizal root colonization on growth and nutrient uptake of two different cowpea (Vigna unguiculata [L.] Walp.) genotypes exposed to drought stress. Emir. J. Food Agric. 21:1-17.

Santalla, M., J.M. Amurrio, A.P. Rodiño and de Ron A.M (2001).Variation in traits affecting nodulation of common bean under intercropping with maize and sole cropping. Euphytica 122:243-255.
Siddiqui, Z.A. and Pichtel J (2008). Mycorrhizae: an overview; In: Z.A. Siddiqui, M.S. Akhtar, K. Futai (Eds). pp. 1-35. Mycorrhizae: Sustainable Agriculture and Forestry. Springer.

Strack, D., T. Fester, B. Hause, W. Schliemann, and Walter M.N (2003). Arbuscular mycorrhiza: Biological, chemical and molecular aspects. J. Chem. Ecol. 29:1955-1979.

Vailleau, F., E. Sartorel, M.-F. Jardinaud, F. Chardon, S. Genin, T. Huguet, L. Gentzbittel, and Petitprez M (2007). Characterization of the interaction between the bacterial wilt pathogen Ralstonia solanacearum and the model legume plant Medicago truncatula. Mol. Plant Microbe Interact. 20:159-167.

Young, N.D., S.B. Cannon, S. Sato, D. Kim, D.R. Cook, C.D. Town, B.A. Roe, and Tabata S (2005). Sequencing the genespaces of Medicago truncatula and Lotus japonicus. Plant Physiol. 137:1174-1181. 